Pacific Journal of Mathematics

PATCH-CONTINUITY OF NORMALIZED RANKS OF
MODULES OVER ONE-SIDED NOETHERIAN RINGS 


\title{
PATCH-CONTINUITY OF NORMALIZED RANKS OF MODULES OVER ONE-SIDED NOETHERIAN RINGS
}

\author{
K. R. GOODEARL
}

For J. T. Stafford's continuity theorem, concerning normalized ranks of finitely generated modules at prime ideals of a noetherian ring, a new proof is developed, which is much simpler than the original proof and also extends the theorem to one-sided noetherian rings.

Introduction. The normalized (reduced) rank at a prime ideal $P$ for a finitely generated right module $A$ over a right noetherian ring $R$ is the rational number

$$
r_{P}(A)=\text { length }\left(A \otimes_{R} Q_{P}\right) / \text { length }\left(Q_{P}\right),
$$

where $Q_{P}$ is the right Goldie quotient ring of $R / P$. In the main result of the paper, we prove that $r_{P}(A)$ is a continuous function of $P$ provided the patch topology is used on the prime spectrum of $R$. A relatively straightforward method of proof is developed, avoiding the technical machinery used by Stafford, to whom the two-sided noetherian case of the theorem is due. If a "generic regularity" condition is assumed, meaning that any element of $R$ regular modulo some prime ideal $P$ remains regular modulo all prime ideals in some patch-neighborhood of $P$, then $r_{P}(A)$ is actually a locally constant function of $P$. This was proved in the two-sided noetherian case by R. B. Warfield, Jr. To put these results in context, we conclude with a brief discussion of some of the known applications.

All rings in this paper are associative with unit, and all modules are unital. This research was partially supported by a National Science Foundation grant.

1. The continuity theorem. A standard result in commutative ring theory is that the local rank of a finitely generated projective module $A$ over a commutative noetherian ring $R$ is a Zariski-continuous function from $\operatorname{Spec}(R)$ to $Z$. The reference to localization can be removed by 
observing that

$$
\operatorname{rank}\left(A_{P}\right)=\operatorname{dim}\left(A_{P} / P A_{P}\right)=\operatorname{dim}\left(A \otimes_{R} Q_{P}\right)
$$

for all $P \in \operatorname{Spec}(R)$, where we have used $Q_{P}$ to denote the quotient field of $R / P$. In the noncommutative case, we are concerned with a finitely generated right module $A$ over a right noetherian ring $R$. Then $Q_{P}$, for $P \in \operatorname{Spec}(R)$, becomes the right Goldie quotient ring of $R / P$, and $\operatorname{dim}\left(A \otimes_{R} Q_{P}\right)$ could be replaced by the composition series length of the right $Q_{P}$-module $A \otimes_{R} Q_{P}$. To obtain a continuity theorem in this setting, we must normalize the length of $A \otimes_{R} Q_{P}$, and we must replace the Zariski topology on $\operatorname{Spec}(R)$ by the patch topology.

Definition. Given any finitely generated module $A$ over a simple artinian ring $Q$, we write $\lambda(A)$ for the normalized length of $A$, that is,

$$
\lambda(A)=\text { length }(A) / \text { length }(Q) .
$$

Note that $\lambda$ is additive on direct sums of $Q$-modules.

Definition. Let $P$ be a prime ideal in a right noetherian ring $R$. For any finitely generated right $R$-module $A$, we set

$$
r_{P}(A)=\lambda\left(A \otimes_{R} Q_{P}\right)=\operatorname{length}\left(A \otimes_{R} Q_{P}\right) / \operatorname{length}\left(Q_{P}\right) .
$$

Note that $r_{P}$ is additive on direct sums of right $R$-modules. Although $r_{P}$ need not be additive on short exact sequences, it is subadditive: namely, if $0 \rightarrow A \rightarrow B \rightarrow C \rightarrow 0$ is any exact sequence of finitely generated right $R$-modules, then $r_{P}(B) \leq r_{P}(A)+r_{P}(C)$, because of the exactness of the sequence

$$
A \otimes_{R} Q_{P} \rightarrow B \otimes_{R} Q_{P} \rightarrow C \otimes_{R} Q_{P} \rightarrow 0 .
$$

Also, since $Q_{P}$ is a flat left $(R / P)$-module, $r_{P}$ is additive on short exact sequences of right $(R / P)$-modules.

Definition. Let $R$ be a ring. For any ideal $I$ of $R$, define

$$
V(I)=\{P \in \operatorname{Spec}(R) \mid P \supseteq I\} \quad \text { and } \quad W(I)=\{P \in \operatorname{Spec}(R) \mid P \nsupseteq I\} .
$$

The sets $V(I)$ are exactly the Zariski-closed subsets of $\operatorname{Spec}(R)$, and the sets $W(I)$ are exactly the Zariski-open subsets. The patch topology on $\operatorname{Spec}(R)$ is the topology with a subbasis of closed sets consisting of all the Zariski-closed sets together with all the Zariski-compact Zariski-open sets $[4, \S \S 2,8]$. 
With the patch topology, $\operatorname{Spec}(R)$ is a compact, Hausdorff, totally disconnected space [4, Proposition 4]. In case $R$ has the ACC on ideals, all the Zariski-open subsets of $\operatorname{Spec}(R)$ are Zariski-compact [2, Proposition 4.3]. Consequently, all the sets $V(I)$ and $W(I)$ are patch-closed and patch-open, and any point $P \in \operatorname{Spec}(R)$ has a patch-neighborhood base consisting of the patch-open sets $V(P) \cap W(I)$ for ideals $I$ properly containing $P$.

Our objective in this section is to prove that for a finitely generated right module $A$ over a right noetherian ring $R$, the rule $P \mapsto r_{P}(A)$ defines a patch-continuous function from $\operatorname{Spec}(R)$ to $\mathbf{Q}$. In case $A$ is projective and $R$ is right fully bounded or of right Krull dimension at most one, Warfield and the author proved that the maps $P \mapsto r_{P}(A)$ are actually locally constant with respect to the patch topology [2, Propositions 4.4 and 4.10]. This result has been extended by Warfield in the two-sided noetherian case to cover non-projective modules, and to allow somewhat more general rings, namely those satisfying the generic regularity condition [13, Lemma 3]. In the meantime, the continuity theorem was proved by Stafford for two-sided noetherian rings [8, Theorem 4.5].

That continuity rather than local constantness is to be expected in general can be seen in the case of the first Weyl algebra over $\mathbf{Z}$. If the ring $R=A_{1}(\mathbf{Z})$ is written as a differential operator ring $\mathbf{Z}[x][\theta ; d / d x]$, and if $A=R / x R$, then $r_{0}(A)=0$ yet $R$ has prime ideals $P(q)$ for all prime integers $q$ such that $\cap P(q)=0$ and $r_{P(q)}(A)=1 / q$ [8, Proposition 7.3]. That the patch topology must be used instead of the Zariski topology can already be seen in the commutative case. For example, let $R=K[x, y]$ be a polynomial ring in two independent indeterminates over a field $K$, and let $A=x R+y R$. Then $r_{M}(A)=1$ for all maximal ideals $M \neq A$, but $r_{A}(A)=2$.

To prove the continuity theorem, we concentrate on the case in which $R$ is a prime right noetherian ring and $A$ is a finitely generated torsion right $R$-module, so that $r_{0}(A)=0$. For any unfaithful subfactor $B$ of $A$, we have $r_{P}(B)=0$ for all $P \in W(\operatorname{ann}(B))$. Hence, the main difficulty occurs with a fully faithful subfactor $C$. To see that $r_{P}(C)$ is arbitrarily small for $P$ in suitable patch-neighborhoods of 0 , we show that all finite direct sums of copies of $C$ are essentially cyclic. The following two lemmas are used to implement this procedure. The first amounts to a simplified analog of the uniqueness of lengths of critical composition series [3, Corollary 2.8; 5, Theorem 3.1]. It may be obtained as an easy consequence of the Schreier Refinement Theorem, or by a short direct argument, as follows. 
Lemma 1.1. Let $A=A_{1} \oplus \cdots \oplus A_{n}$ where $A_{1}, \ldots, A_{n}$ are $\alpha$-critical modules ( for some ordinal $\alpha$ ), and let

$$
B_{0}=0 \subset B_{1} \subset B_{2} \subset \cdots \subset B_{n}
$$

be a chain of $n+1$ submodules of $A$. If $\mathrm{K} \cdot \operatorname{dim}\left(B_{i} / B_{i-1}\right)=\alpha$ for each $i=1, \ldots, n$, then $B_{n}$ is essential in $A$.

Proof. As $A_{1}$ is uniform, the case $n=1$ is trivial. Now let $n>1$, and assume that the lemma holds for direct sums of fewer $\alpha$-critical modules.

If $B_{n} \cap A_{n}=0$, the projection $p: A \rightarrow A_{1} \oplus \cdots \oplus A_{n-1}$ maps $B_{n}$ isomorphically into $A_{1} \oplus \cdots \oplus A_{n-1}$. By the induction hypothesis, $p\left(B_{n-1}\right)$ is essential in $A_{1} \oplus \cdots \oplus A_{n-1}$, and so $p\left(B_{n-1}\right) \cap A_{i} \neq 0$ for $i=1, \ldots, n-1$. Then $A_{i} /\left(p\left(B_{n-1}\right) \cap A_{l}\right)$ has Krull dimension less than $\alpha$ for each $i=1, \ldots, n-1$, whence

$$
\mathrm{K} \cdot \operatorname{dim}\left(\left(A_{1} \oplus \cdots \oplus A_{n-1}\right) / p\left(B_{n-1}\right)\right)<\alpha .
$$

But then $\mathrm{K} \cdot \operatorname{dim}\left(p\left(B_{n}\right) / p\left(B_{n-1}\right)\right)<\alpha$, contradicting our hypotheses.

Thus $B_{n} \cap A_{n} \neq 0$, and similarly $B_{n} \cap A_{j} \neq 0$ for each $j=1, \ldots, n$. Then each $B_{n} \cap A_{j}$ is essential in $A_{j}$, and therefore $B_{n}$ is essential in $A$.

Definition. A fully faithful module is any nonzero module all of whose nonzero submodules are faithful. (In contrast, a completely faithful module is a nonzero module all of whose nonzero subfactors are faithful.)

Definition. An $\alpha$-homogeneous module (for some ordinal $\alpha$ ) is any nonzero module all of whose nonzero submodules have Krull dimension $\alpha$.

The following lemma was inspired by the result of Eisenbud and Robson that all completely faithful modules of finite length are cyclic [1, Lemma 3.1], and by the result of Stafford that over a ring of Krull dimension greater than $n$, all completely faithful noetherian modules of Krull dimension $n$ can be generated by $n+1$ elements [7, Theorem 1.3].

LEMMA 1.2. Let $R$ be a right noetherian ring, and let $A$ be a finitely generated, fully faithful, $\alpha$-homogeneous right $R$-module, for some ordinal $\alpha$. If r.K.dim $(R)>\alpha$, then $A$ has an essential cyclic submodule.

Proof. Since $A$ may be replaced by an essential submodule, there is no loss of generality in assuming that $A=A_{1} \oplus \cdots \oplus A_{n}$ for some $\alpha$-critical submodules $A_{i}$. 
We claim that there are elements $x_{i} \in A_{i}$ for each $i=1, \ldots, n$ such that if $I_{0}=R$ and $I_{j}=\operatorname{ann}_{R}\left(\left\{x_{1}, \ldots, x_{j}\right\}\right)$ then $K \cdot \operatorname{dim}\left(I_{j-1} / I_{j}\right)=\alpha$ for each $j=1, \ldots, n$. First choose any nonzero element $x_{1} \in A_{1}$, and observe that $I_{0} / I_{1}$ has Krull dimension $\alpha$ because $A_{1}$ is $\alpha$-critical.

Now suppose that $x_{1}, \ldots, x_{j}$ have been chosen, for some $j<n$. Since

$$
\mathrm{K} \cdot \operatorname{dim}\left(R / I_{j}\right)=\alpha<\mathrm{r} \cdot \mathrm{K} \cdot \operatorname{dim}(R),
$$

we must have $I_{j} \neq 0$. As $A$ is fully faithful, there exists $x_{j+1} \in A_{j+1}$ such that $x_{j+1} I_{j} \neq 0$, and

$$
\mathrm{K} \cdot \operatorname{dim}\left(I_{j} / I_{j+1}\right)=\mathrm{K} \cdot \operatorname{dim}\left(x_{j+1} I_{j}\right)=\alpha .
$$

This completes the proof of the claim.

Set $x=x_{1}+\cdots+x_{n}$, and observe that $\operatorname{ann}_{R}(x)=I_{n}$, whence

$$
x I_{j-1} / x I_{j} \simeq I_{j-1} / I_{j}
$$

for each $j=1, \ldots, n$. Thus $x R$ has a chain

$$
x I_{0}=x R \supset x I_{1} \supset \cdots \supset x I_{n}=0
$$

of $n+1$ submodules in which the successive factors $x I_{j-1} / x I_{j}$ all have Krull dimension $\alpha$. By Lemma 1.1, $x R$ is essential in $A$.

Proposition 1.3. Let $R$ be a prime right noetherian ring, let $A$ be a finitely generated torsion right $R$-module, and let $\varepsilon$ be a positive real number. Then $R$ contains a nonzero ideal I such that $r_{P}(A)<\varepsilon$ for all prime ideals $P$ not containing $I$.

Proof. Set $\alpha=\mathrm{K} \cdot \operatorname{dim}(A)$, and note that the case $\alpha=-1$ is trivial. Now let $\alpha \geq 0$, and assume that the proposition holds for finitely generated torsion modules of Krull dimension less than $\alpha$. There exists a chain

$$
A_{0}=0 \subset A_{1} \subset \cdots \subset A_{m}=A
$$

of submodules of $A$ in which the successive subfactors are all critical. Since the functions $r_{P}$ are subadditive, it suffices to find nonzero ideals $I_{1}, \ldots, I_{m}$ in $R$ such that $r_{P}\left(A_{j} / A_{j-1}\right)<\varepsilon / m$ for all prime ideals $P \nsupseteq I_{j}$. (Take $I=I_{1} I_{2} \cdots I_{m}$.) Thus we may assume that $A$ is $\alpha$-critical.

First suppose that $A$ contains a nonzero unfaithful submodule $B$, and set $I_{1}=\operatorname{ann}_{R}(B)$. If $P$ is any prime ideal not containing $I_{1}$, then $B / B P$ is an unfaithful $(R / P)$-module and so is torsion over $R / P$, whence $r_{P}(B)=0$. As $\mathrm{K} \cdot \operatorname{dim}(A / B)<\alpha$, there exists a nonzero ideal $I_{2}$ in $R$ such that $r_{P}(A / B)<\varepsilon$ for all prime ideals $P \nsubseteq I_{2}$. Thus $r_{P}(A)<\varepsilon$ for all prime ideals $P \nsupseteq I_{1} I_{2}$. 
Finally, suppose that $A$ is fully faithful, and note that because $A$ is a torsion module, r.K.dim $(R)>\alpha$. Choose a positive integer $n>2 / \varepsilon$. Then $A^{n}$ is a fully faithful $\alpha$-homogeneous right $R$-module, and so by Lemma 1.2 it has an essential cyclic submodule $C$. Since $A$ is $\alpha$-critical, $\mathrm{K} \cdot \operatorname{dim}\left(A^{n} / C\right)<\alpha$. Hence, $R$ contains a nonzero ideal $I$ such that $r_{P}\left(A^{n} / C\right)<1$ for all prime ideals $P \nsupseteq I$. For such $P$, we have $r_{P}(C) \leq 1$ because $C$ is cyclic, whence $r_{P}\left(A^{n}\right)<2$. Therefore $r_{P}(A)<2 / n<\varepsilon$ for all prime ideals $P \nsupseteq I$.

A. V. Jategaonkar has pointed out that the use of Krull dimension in proving Proposition 1.3 is not essential, for monoform modules can be used in place of critical modules. A proof along such lines is to be incorporated in [6].

THEOREM 1.4. Let $A$ be a finitely generated right module over a right noetherian ring $R$. Then the rule $P \mapsto r_{P}(A)$ defines a patch-continuous function from $\operatorname{Spec}(R)$ to $\mathbf{Q}$.

Proof. Given a prime ideal $T$ in $R$ and a positive real number $\varepsilon$, we must find an ideal $I$ properly containing $T$ such that

$$
\left|r_{P}(A)-r_{T}(A)\right|<\varepsilon
$$

for all prime ideals $P \in V(T) \cap W(I)$. As $r_{P}(A)=r_{P / T}(A / A T)$ for all $P \in V(T)$, we may reduce to the case that $T=0$, without loss of generality.

We first find a nonzero ideal $I_{1}$ such that $r_{P}(A)<r_{0}(A)+\varepsilon$ for all $P \in W\left(I_{1}\right)$. Write $r_{0}(A)=k / n$ for some $k \in \mathbf{Z}^{+}$and $n \in \mathbf{N}$. Then $r_{0}\left(A^{n}\right)=k$, and hence $A^{n} \otimes_{R} Q_{0}$ is a free right $Q_{0}$-module of rank $k$. Choose a basis $\left\{x_{1}, \ldots, x_{k}\right\}$ for $A^{n} \otimes_{R} Q_{0}$, and write each $x_{i}=a_{i} \otimes c^{-1}$ where $a_{1}, \ldots, a_{k} \in A^{n}$ and $c$ is a regular element in $R$. Set $B=$ $a_{1} R+\cdots+a_{k} R$, and observe that $\left(A^{n} / B\right) \otimes_{R} Q_{0}=0$, whence $A^{n} / B$ is a torsion module. By Proposition 1.3, there exists a nonzero ideal $I_{1}$ in $R$ such that $r_{P}\left(A^{n} / B\right)<n \varepsilon$ for all $P \in W\left(I_{1}\right)$. Since $B$ is generated by $k$ elements, $r_{P}(B) \leq k$ for all prime ideals $P$. For $P \in W\left(I_{1}\right)$, we thus have $r_{P}\left(A^{n}\right)<k+n \varepsilon$, and hence

$$
r_{P}(A)<(k / n)+\varepsilon=r_{0}(A)+\varepsilon .
$$

Choose an exact sequence $0 \rightarrow K \rightarrow F \rightarrow A \rightarrow 0$ of right $R$-modules such that $F$ is free of finite rank $m$, and note that

$$
m=r_{0}(F)=r_{0}(K)+r_{0}(A) \text {. }
$$


Applying the argument of the previous paragraph to the module $K$, we obtain a nonzero ideal $I_{2}$ in $R$ such that $r_{P}(K)<r_{0}(K)+\varepsilon$ for all $P \in W\left(I_{2}\right)$. As

$$
m=r_{P}(F) \leq r_{P}(K)+r_{P}(A)
$$

for any such $P$, we conclude that

$$
r_{P}(A) \geq m-r_{P}(K)>m-r_{0}(K)-\varepsilon=r_{0}(A)-\varepsilon
$$

for all $P \in W\left(I_{2}\right)$.

Therefore, $\left|r_{P}(A)-r_{0}(A)\right|<\varepsilon$ for all $P \in W\left(I_{1} I_{2}\right)$.

2. Generic regularity. In those cases where the continuity theorem can be improved to say that the maps $P \mapsto r_{P}(A)$ are locally constant with respect to the patch topology, regularity modulo prime ideals defines open sets in the patch topology. Namely, consider a right noetherian ring $R$, a prime ideal $T$ of $R$, and an element $x \in \mathscr{C}(T)$, that is, $x \in R$ and $x+T$ is a regular element of $R / T$. Then $(x R+T) / T$ is an essential right ideal of $R / T$ and $R /(x R+T)$ is a torsion right $(R / T)$-module, so that $r_{T}(R / x R)=0$. If the map $P \mapsto r_{P}(R / x R)$ is locally constant in the patch topology, the set

$$
X=\left\{P \in \operatorname{Spec}(R) \mid r_{P}(R / x R)=0\right\}
$$

is patch-open. For all $P \in X$, observe that $R /(x R+P)$ is a torsion right $(R / P)$-module and so $(x R+P) / P$ is an essential right ideal of $R / P$, whence $x \in \mathscr{C}(P)$. Since $x$ is regular modulo all the prime ideals in the patch-open set $X$, we may say that $x$ is "generically" regular (with respect to the patch topology).

Conversely, we shall see that generic regularity implies patch-localconstantness of the maps $P \mapsto r_{P}(A)$.

Definition. Let $R$ be a ring. A subset $X$ of $\operatorname{Spec}(R)$ satisfies the generic regularity condition (or is sparse, in the terminology of [6]) provided that for any prime ideal $T$ of $R$ (not necessarily in $X$ ) and any element $x \in \mathscr{C}(T)$, there is a patch-neighborhood $U$ of $T$ such that $x \in \mathscr{C}(P)$ for all $P \in X \cap U$. In other words, there must exist an ideal $I$ properly containing $T$ such that $x \in \mathscr{C}(P)$ for those $P \in X$ such that $P \supseteq T$ but $P \nsupseteq I$. 
Two easy cases in which generic regularity occurs were observed in [6, Chapter 6] and in [13, Lemma 1], namely any set of completely prime ideals in any right noetherian ring, and any set of prime ideals in any right fully bounded right noetherian ring satisfy generic regularity. Another easy case is given in the following proposition.

Proposition 2.1. Let $R$ be a right noetherian ring with $\mathrm{r} \cdot \mathrm{K} \cdot \operatorname{dim}(R) \leq 1$. Then any subset of $\operatorname{Spec}(R)$ satisfies the generic regularity condition.

Proof. It suffices to show that generic regularity holds for $\operatorname{Spec}(R)$. Consider $T \in \operatorname{Spec}(R)$ and $x \in \mathscr{C}(T)$, and note that $(x R+T) / T$ is an essential right ideal of $R / T$. If $T$ is a maximal ideal of $R$, then $\{T\}$ is a patch-neighborhood of $T$ and we are done. Suppose then that $T$ is not a maximal ideal. As r.K.dim $(R / T) \leq 1$, all proper prime factor rings of $T$ and all finitely generated torsion right $(R / T)$-modules are artinian. In particular, $R /(x R+T)$ must have finite length. Hence, there are at most finitely many maximal ideals $M_{i} \supset T$ such that the (unique) simple right $\left(R / M_{i}\right)$-module appears as a composition factor of $R /(x R+T)$. There is an ideal $I$ properly containing $T$ such that each $M_{i} \supseteq I$.

Let $P \in V(T) \cap W(I)$. Since $x \in \mathscr{C}(T)$ to begin with, suppose that $P \neq T$. Then $P$ is a maximal ideal of $R$. If $A$ is any composition factor of $R /(x R+T)$, the annihilator of $A$ is either $T$ or one of the $M_{i}$. In either case, $P \neq \operatorname{ann}_{R}(A)$ and so $P \nsubseteq \operatorname{ann}_{R}(A)$, whence $A P=A$ and $r_{P}(A)=0$. As a result, $r_{P}(R /(x R+T))=0$ and hence $R /(x R+P)$ is a torsion right $(R / P)$-module. Therefore $x \in \mathscr{C}(P)$.

As mentioned above, any set $X$ of completely prime ideals in a right noetherian ring $R$ (in other words, the right Goldie rank of $R / P$ is 1 for each $P \in X$ ) is easily seen to satisfy the generic regularity condition. More generally, any set $X$ of prime ideals of $R$ such that there is a bound on the Goldie ranks of the prime factor rings $R / P$ for $P \in X$ must satisfy generic regularity. In case $R$ is noetherian on both sides, this follows from Stafford's continuity theorem [10, first proof of Corollary 3.9; 13, Lemma 1]. Stafford and Snider devised an ultraproduct argument to prove this result for right noetherian rings $[10$, second proof of Corollary $3.9 ; 6$, Chapter 6], but with the continuity theorem available for right noetherian rings, it is much more direct to use that, as follows.

Proposition 2.2. Let $R$ be a right noetherian ring and $X$ a subset of $\operatorname{Spec}(R)$. If there exists a positive integer $n$ such that $R / P$ has right Goldie rank at most $n$ for each $P \in X$, then $X$ satisfies the generic regularity condition. 
Proof. For any $P \in X$, the quotient ring $Q_{P}$ of $R / P$ has length at most $n$, and so $\lambda(A) \geq 1 / n$ for all nonzero finitely generated right $Q_{P}$-modules $A$. Consequently, $r_{P}(B)$, for any finitely generated right $R$-module $B$, is either 0 or at least $1 / n$.

Given $T \in \operatorname{Spec}(R)$ and $x \in \mathscr{C}(T)$, we have $r_{T}(R / x R)=0$. By Theorem 1.4, there exists a patch-neighborhood $U$ of $T$ such that $r_{P}(R / x R)<1 / n$ for all $P \in U$. Thus for $P \in X \cap U$, we have $r_{P}(R / x R)=0$, and therefore $x \in \mathscr{C}(P)$.

Without a bound on the Goldie ranks of prime factor rings, generic regularity need not hold. For an example, consider again

$$
R=A_{1}(\mathbf{Z})=\mathbf{Z}[x][\theta ; d / d x] .
$$

Since $R$ is a domain, $x \in \mathscr{C}(0)$, yet $R$ has prime ideals $P(q)$ for all prime integers $q$ such that $\cap P(q)=0$ and $x \notin \mathscr{C}(P(q))$ [8, Proposition 7.3]. Thus $\operatorname{Spec}(R)$ does not satisfy the generic regularity condition.

That generic regularity implies patch-local-constantness of the maps $P \mapsto r_{P}(A)$ over right and left noetherian rings was shown by Warfield in [13, Lemma 3]. For the right noetherian case, a modification of the proof of Theorem 1.4 may be used, as follows.

THEOREM 2.3. Let $A$ be a finitely generated right module over a right noetherian ring $R$, and let $X$ be a subset of $\operatorname{Spec}(R)$ that satisfies the generic regularity condition. Then any prime ideal $T$ of $R$ has a patch-neighborhood $U$ such that $r_{P}(A)=r_{T}(A)$ for all $P \in X \cap U$.

Proof. We first prove the analog of Proposition 1.3, namely the case that $r_{T}(A)=0$.

Choose generators $a_{1}, \ldots, a_{n}$ for $A$. Since $r_{T}(A)=0$, the module $A / A T$ is torsion as a right $(R / T)$-module, and hence there exists $x \in$ $\mathscr{C}(T)$ such that $a_{i} x \in A T$ for $i=1, \ldots, n$. By generic regularity, $T$ has a patch-neighborhood $U$ such that $x \in \mathscr{C}(P)$ for all $P \in X \cap U$. Since $U$ may be intersected with $V(T)$, there is no loss of generality in assuming that $U \subseteq V(T)$. Now for any $P \in X \cap U$ we have $x \in \mathscr{C}(P)$ and $a_{i} x \in$ $A T \subseteq A P$ for $i=1, \ldots, n$, whence $A / A P$ is a torsion right $(R / P)$-module, and thus $r_{P}(A)=0$.

With this case in hand, we follow the proof of Theorem 1.4, replacing $[<\varepsilon]$ everywhere by $[=0]$, and ignoring prime ideals not in $X$.

Theorem 2.3 simultaneously generalizes [2, Propositions 4.4 and 4.10]. 
3. Applications. The main applications of the original patch-localconstantness and patch-continuity results concerned estimates for numbers of generators of modules over noetherian rings $[11,12,8,9]$ and the identification of extremal states on $K_{0}$ of noetherian rings [2, 8]. To spotlight the uses of local constantness and continuity arguments, we briefly indicate their occurrence in some of these results.

(a) By analogy with the Forster-Swan Theorem, one wishes to estimate the number of generators required for a finitely generated right module $A$ over a right noetherian ring $R$, in terms of the minimal number of generators $g(P, A)$ for the $Q_{P}$-module $A \otimes_{R} Q_{P}$, for each prime ideal $P$, or for each $J$-prime ideal $P$. Observe that $g(P, A)$ is the smallest integer greater than or equal to $r_{P}(A)$. If $g(P, A)=0$, set $b(P, A)=0$, while if $g(P, A) \neq 0$, set

$$
b(P, A)=g(P, A)+J-\operatorname{dim}(R / P) .
$$

In case $R$ is right fully bounded, Warfield proved that $A$ can be generated by

$$
b=\sup \{b(P, A) \mid P \in J-\operatorname{Spec}(R)\}
$$

elements [11, Theorem B; 12, Theorems C, 2].

A key reduction step in Warfield's proof requires that, assuming $b$ to be nonzero and finite, only finitely many $J$-prime ideals $P$ satisfy $b(P, A)$ $=b[11$, Lemma $1 ; 12$, Lemma 5]. The proofs of these lemmas used patch-local-constantness together with an induction on $J$-dimension. There is a more direct argument using compactness, which was communicated to the author by Warfield, as follows.

By [2, Proposition 4.3], $J-\operatorname{Spec}(R)$ is patch-closed in $\operatorname{Spec}(R)$ and so is patch-compact. Using local constantness, each $P \in J-\operatorname{Spec}(R)$ has a patch-neighborhood $U(P)$ of the form $V(P) \cap W\left(I_{P}\right)$ such that $r_{Q}(A)=$ $r_{P}(A)$ for all $Q \in U(P)$. Then $g(Q, A)=g(P, A)$ for such $Q$. If $Q \neq P$, then $Q$ properly contains $P$, and so if $Q \in J-\operatorname{Spec}(R)$ we obtain $J-\operatorname{dim}(R / Q)<J-\operatorname{dim}(R / P)$ and hence $b(Q, A)<b$. By compactness, some $U\left(P_{1}\right), \ldots, U\left(P_{n}\right)$ cover $J$-Spec $(R)$, and any $J$-prime ideals $P$ which satisfy $b(P, A)=b$ must be among $P_{1}, \ldots, P_{n}$.

(b) Warfield also proved that if $R$ is right fully bounded and noetherian on both sides, $A$ can be generated by

$$
\max \{g(M, A) \mid M \text { is a maximal ideal of } R\}+J-\operatorname{dim}(R)
$$

elements [12, Theorems A, 5]. This estimate follows from the previous one provided that for each $J$-prime ideal $P$ there is a maximal ideal $M$ such 
that $g(P, A) \leq g(M, A)[12$, Lemma 6]. This is an easy consequence of local constantness, as follows.

There is an ideal $I$ properly containing $P$ such that $r_{Q}(A)=r_{P}(A)$ for all $Q$ in $V(P) \cap W(I)$. Since $P$ is $J$-prime, there is a right primitive ideal $M \supseteq P$ that does not contain $I$. Then $r_{M}(A)=r_{P}(A)$ and so $g(P, A)=g(M, A)$. As $R$ is right fully bounded, $M$ is a maximal ideal. (Because the prime spectrum of any right fully bounded right noetherian ring satisfies generic regularity, the left noetherian hypothesis for Warfield's second estimate is not needed. Both estimates can be proved assuming only that $R$ is right noetherian, all right primitive factor rings of $R$ are artinian, and $J-\operatorname{Spec}(R)$ satisfies generic regularity.)

(c) Stafford's number of generator estimates apply to any finitely generated right module $A$ over any right and left noetherian ring $R$. In these estimates the value $b(P, A)$ is either 0 or $g(P, A)+\mathrm{r} \cdot \mathrm{K} \cdot \operatorname{dim}(R / P)$, and $A$ can be generated by

$$
\max \{\mathrm{K} \cdot \operatorname{dim}(A)+1, \sup \{b(P, A) \mid P \in J-\operatorname{Spec}(R)\}\}
$$

elements [8, Theorem 3.1]. Stafford also proved that $A$ can be generated by

$\max \{g(P, A) \mid P$ is a right primitive ideal of $R\}+\mathrm{r} \cdot \mathrm{K} \cdot \operatorname{dim}(R)$

elements [8, Corollary 4.6]. The second estimate follows from the first provided that for each $J$-prime ideal $P$ there is a right primitive ideal $M$ such that $g(P, A) \leq g(M, A)$, and Stafford obtained this from patch-continuity, as follows.

Since $g(P, A)$ is the smallest integer greater than or equal to $r_{P}(A)$, we have $r_{P}(A)>g(P, A)-1$. By patch-continuity, there is an ideal $I$ properly containing $P$ such that $r_{Q}(A)>g(P, A)-1$ for all $Q$ in $V(P) \cap W(I)$. Since $P$ is $J$-prime, there is a right primitive ideal $M \supseteq P$ that does not contain $I$. Then $g(M, A) \geq r_{M}(A)>g(P, A)-1$, and hence $g(M, A) \geq g(P, A)$, because these are integers.

(d) The state space of $K_{0}$ of a ring $R$ is a compact convex subset $S t(R)$ of the product space of all real-valued functions on $K_{0}(R)$, consisting of all group homomorphisms $s: K_{0}(R) \rightarrow \mathbf{R}$ such that $s([R])$ $=1$ and $s([A]) \geq 0$ for all finitely generated projective right $R$-modules $A$. In case $R$ is right noetherian, each of the normalized rank functions $r_{P}$ (for $P \in \operatorname{Spec}(R)$ ) induces a state $s_{P} \in \operatorname{St}(R)$, where $s_{P}([A])=r_{P}(A)$ for all $A$. 
Warfield and the author proved that for a right noetherian ring $R$ such that either $R$ is right fully bounded with finite $J$-dimension, or r.K.dim $(R) \leq 1$, every extreme point of $S t(R)$ has the form $s_{P}$ for some $P \in J-\operatorname{Spec}(R)$ [2, Theorems 4.5, 4.11]. Stafford derived the same conclusion when $R$ is right and left noetherian with finite right Krull dimension [8, Theorem 6.4]. A key step in these proofs is to show that $\left\{s_{p} \mid P \in\right.$ $J$-Spec $(R)\}$ is a compact subset of $\operatorname{St}(R)$. This follows from the patchcompactness of $J-\operatorname{Spec}(R)$, together with the patch-continuity of the map $P \mapsto s_{P}$, which is an immediate consequence of the patch-continuity of the maps $P \mapsto r_{P}(A)$.

\section{REFERENCES}

[1] D. Eisenbud and J. C. Robson, Modules over Dedekind prime rings, J. Algebra, 16 (1970), 67-85.

[2] K. R. Goodearl and R. B. Warfield, Jr., State spaces of $K_{0}$ of noetherian rings, J. Algebra, 71 (1981), 322-378.

[3] R. Gordon, Gabriel and Krull dimension, in Ring Theory, Proceedings of the Oklahoma Conference (B. R. McDonald, A. R. Magid, and K. C. Smith, Eds.), pp. 241-295, New York (1974) Dekker.

[4] M. Hochster, Prime ideal structure in commutative rings, Trans. Amer. Math. Soc., 142 (1969), 43-60.

[5] A. V. Jategaonkar, Jacobson's conjecture and modules over fully bounded noetherian rings, J. Algebra, 30 (1974), 103-121.

[6] Localization in Noetherian Rings, London Math. Soc. Lecture Notes, (to appear).

[7] J. T. Stafford, Completely faithful modules and ideals of simple noetherian rings, Bull. London Math. Soc., 8 (1976), 168-173.

[8] - Generating modules efficiently: algebraic $K$-theory for noncommutative noetherian rings, J. Algebra, 69 (1981), 312-346.

[9] Generating modules efficiently over noncommutative rings, in Seminaire d'Algèbre P. Dubreil et M.-P. Malliavin, pp. 72-88, Lecture Notes in Math., No. 924, Berlin (1982) Springer-Verlag.

[10] The Goldie rank of a module, Proc. Conf. Noetherian Rings Oberwolfach 1983 (to appear).

[11] R. B. Warfield, Jr., Modules over fully bounded noetherian rings, in Ring Theory Waterloo 1978 (D. Handelman and J. Lawrence, Eds.), pp. 339-352, Lecture Notes in Math., No. 734, Berlin (1979) Springer-Verlag.

[12] _ The number of generators of a module over a fully bounded ring, J. Algebra, 66 (1980), 425-447.

[13] , Noncommutative localized rings, Seminaire d'Algèbre P. Dubreil et M.-P. Malliavin (to appear).

Received August 30, 1984 and in revised form January 25, 1985.

UNIVERSITY OF UTAH

Salt Lake City, UT 84112 


\section{PACIFIC JOURNAL OF MATHEMATICS EDITORS}

\author{
V. S. VARADARAJAN (Managing Editor) \\ University of California \\ Los Angeles, CA 90024 \\ Hebert Clemens \\ University of Utah \\ Salt Lake City, UT 84112 \\ Charles R. DePrima \\ California Institute of Technology \\ Pasadena, CA 91125
}

R. FINN

Stanford University

Stanford, CA 94305

HermanN FlaschKa

University of Arizona

Tucson, AZ 85721

RAMESH A. GANGOLlI

University of Washington

Seattle, WA 98195

ROBION KIRBY

University of California

Berkeley, CA 94720

ASSOCIATE EDITORS
R. ARENS
E. F. BECKENBACH
B. H. NEUMANN
F. WOLF
K. YoshidA (1906-1982)

C. C. Moore

University of California

Berkeley, CA 94720

H. SAMELSON

Stanford University

Stanford, CA 94305

HAROLD STARK

University of California, San Diego

La Jolla, CA 92093

\section{SUPPORTING INSTITUTIONS}

UNIVERSITY OF ARIZONA

UNIVERSITY OF BRITISH COLUMBIA

CALIFORNIA INSTITUTE OF TECHNOLOGY

UNIVERSITY OF CALIFORNIA

MONTANA STATE UNIVERSITY

UNIVERSITY OF NEVADA, RENO

NEW MEXICO STATE UNIVERSITY

OREGON STATE UNIVERSITY
UNIVERSITY OF OREGON

UNIVERSITY OF SOUTHERN CALIFORNIA

STANFORD UNIVERSITY

UNIVERSITY OF HAWAII

UNIVERSITY OF TOKYO

UNIVERSITY OF UTAH

WASHINGTON STATE UNIVERSITY

UNIVERSITY OF WASHINGTON 


\section{Pacific Journal of Mathematics}

Vol. 122, No. $1 \quad$ January, 1986

Michael James Cambern, Near isometries of Bochner $L^{1}$ and $L^{\infty}$ spaces ....1 Kun Soo Chang, Gerald William Johnson and David Lee Skoug, The

Feynman integral of quadratic potentials depending on two time

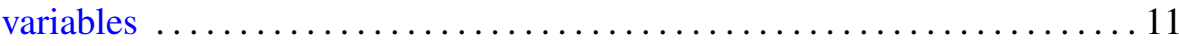

Robert Coleman, One-dimensional algebraic formal groups $\ldots \ldots \ldots \ldots \ldots 35$

Alberto Collino, The Abel-Jacobi isomorphism for the cubic fivefold .......43

N. J. Dev and S. S. Khare, Finite group action and vanishing of $N_{*}^{G}[F] \ldots 57$

Harold George Diamond and Jeffrey D. Vaaler, Estimates for partial sums

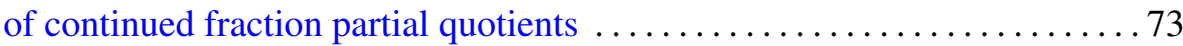

Kenneth R. Goodearl, Patch-continuity of normalized ranks of modules

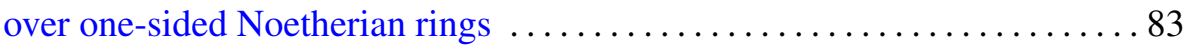

Dean Robert Hickerson and Sherman K. Stein, Abelian groups and

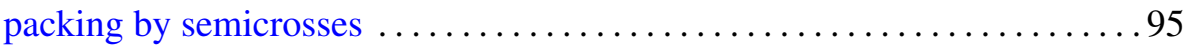

Karsten Johnsen and Harmut Laue, Fitting structures $\ldots \ldots \ldots \ldots \ldots 11$

Darren Long, Discs in compression bodies . ................... 129

Joseph B. Miles, On the growth of meromorphic functions with radially distributed zeros and poles ........................... 147

Walter Volodymyr Petryshyn, Solvability of various boundary value problems for the equation $x^{\prime \prime}=f\left(t, x, x^{\prime}, x^{\prime \prime}\right)-y \ldots \ldots \ldots \ldots \ldots . \ldots 169$

Elżbieta Pol, The Baire-category method in some compact extension problems

Masami Sakai, A new class of isocompact spaces and related results 211

Thomas Richard Shemanske, Representations of ternary quadratic forms and the class number of imaginary quadratic fields ..

Tsuyoshi Uehara, On class numbers of cyclic quartic fields 